\title{
Improved source path localisation in ring applicators and the clinical impact for gynecological brachytherapy
}

\author{
Irene Humer, ScD', Prof. Christian Kirisits, ScD',2, Daniel Berger, PhD', Petra Trnková, PhD', Prof. Richard Pötter, MD',2 \\ Nicole Nesvacil, ScD 1,2 \\ 'Department of Radiotherapy, Comprehensive Cancer Center, Medical University of Vienna, ${ }^{2}$ Christian Doppler Laboratory for Medical \\ Radiation Research for Radiation Oncology, Vienna, Austria
}

\begin{abstract}
Purpose: The path of subsequent dwell positions of an afterloader source being moved through a ring applicator for cervix cancer brachytherapy deviates from an ideal circle and the position of marker wires. This can lead to deviations of several millimetres between real and assumed dwell positions for treatment planning with simplified source path models. The aim of this study was to test video- and autoradiography-based methods for source path determination, and to study the influence of dwell position accuracy on dose-volume histogram (DVH)-parameters.

Material and methods: Videos of the exact motion of the source wire through three different $(r=26,30,34 \mathrm{~mm})$ computed tomography/magnetic resonance (CT/MR) compatible plastic ring applicators were recorded. Observed dwell positions covering the whole length of each applicators channel were used to adjust the circular source path model. The agreement of the true source positions derived from video analysis with those of the corrected circular source path was verified using autoradiography. The impact of an accurate source path definition on dose planning was analysed by simulating clinically relevant uncertainties in 10 clinical treatment plans.

Results: Depending on the ring size, source path diameters had to be increased by $0.5-1.0 \mathrm{~mm}$ in order to achieve acceptable maximum differences between observed and corrected dwell positions (1.3-2.0 mm). Autoradiography analysis showed a positional accuracy within $\pm 3 \mathrm{~mm}$ (extended standard deviation $k=2$ ). For shifts of $\pm 2.5 \mathrm{~mm}$ for even all dwell positions, the systematic and random variation of the $\mathrm{D}_{2 \mathrm{~cm}^{3}}$ for bladder, rectum, and sigmoid was within $3 \%$, while the impact on DVH uncertainties was much smaller for clinical target volume (CTV) $)_{\mathrm{HR}}$ and gross tumour volume (GTV).

Conclusions: It is strongly advised to verify the real source path for ring applicators during acceptance testing in order to assure accurate source path definition and dose planning. Autoradiography can be used for source path verification with acceptable accuracy for treatment planning and dose reporting.
\end{abstract}

Key words: applicator geometry, brachytherapy, gynaecological, quality assurance, source path reconstruction, treatment planning.

\section{Purpose}

During the last decade, image based adaptive brachytherapy has been implemented in an increasing number of institutions, and improved the precision of dose delivery and dose reporting for target volumes as well as organs at risk (OARs) [1]. A substantial reduction of uncertainties can lead to an increase of local control as well as a reduction of side effects. A review of clinical brachytherapy uncertainties has recently been published by Kirisits et al. [2]. Geometric uncertainties in cervix cancer brachytherapy originating from afterloader source positioning as well as reconstruction of the applicator have been discussed in various papers [3]. Tanderup et al. summarized the estimated dose changes to target volumes and OAR of about $4 \%$ due to dwell position uncertainties [4]. Concerning the dosimetric effect of applicator displacement, for instance, they found significant changes in dose to some organs at risk (rectum and bladder) in the case of cranio-caudal applicator shifts. Some vendors provide additional tools with libraries of 3D applicators for easier reconstruction of the tandem and ring, e.g. by 3-point-positioning and further manual fine-tuning by translation and rotation [3], while basic versions
Address for correspondence: Nicole Nesvacil, DSc, Department of Radiotherapy, Medical University Vienna/AKH Vienna Währinger Gürtel 18-20, A-1090 Vienna, Austria, phone: +43 140400/26950, fax: +43 140400/2693, e-mail: nicole.nesvacil@meduniwien.ac.at
Received: 03.06 .2015

Accepted: 10.06.2015

Published: 25.06.2015 
of planning systems require direct reconstruction of the source path inside an applicator. In addition to minimizing the uncertainties introduced by applicator shifts in all directions [4], precise dose calculation based on any 3D imaging modality and planning system requires to know the exact position of the actual source path inside the applicator. For the ring applicator, this source path was usually described by a circle with a nominal diameter, the location of inserted marker wires visible in computed tomography (CT) or radiographs or contrast material visible in magnetic resonance imaging (MR). However, the actual source path may deviate from an ideal circle, the marker wire or contrast material location.

Recently, Awunor et al. presented a method for the source position reconstruction in ring applicators with the aid of gafchromic films. They found shifts up to $6.1 \mathrm{~mm}$ between expected and measured positions [5].

Systematic deviations of the clinically relevant dwell positions (see standard loading patterns [6]) between the nominal and the real source path may lead to systematic over- or underestimation of doses delivered to the target and OAR in the vicinity of the ring applicator. So far, there is no evidence in literature of a systematic analysis of the dosimetric effect which reconstruction of the actual location of the source path inside a ring applicator's source channel may have on target structures and OARs.

In contrast to interstitial applicators, such as needles or tubes, the source movement in ring applicators does not follow a simple circular pathway. In principle, after-

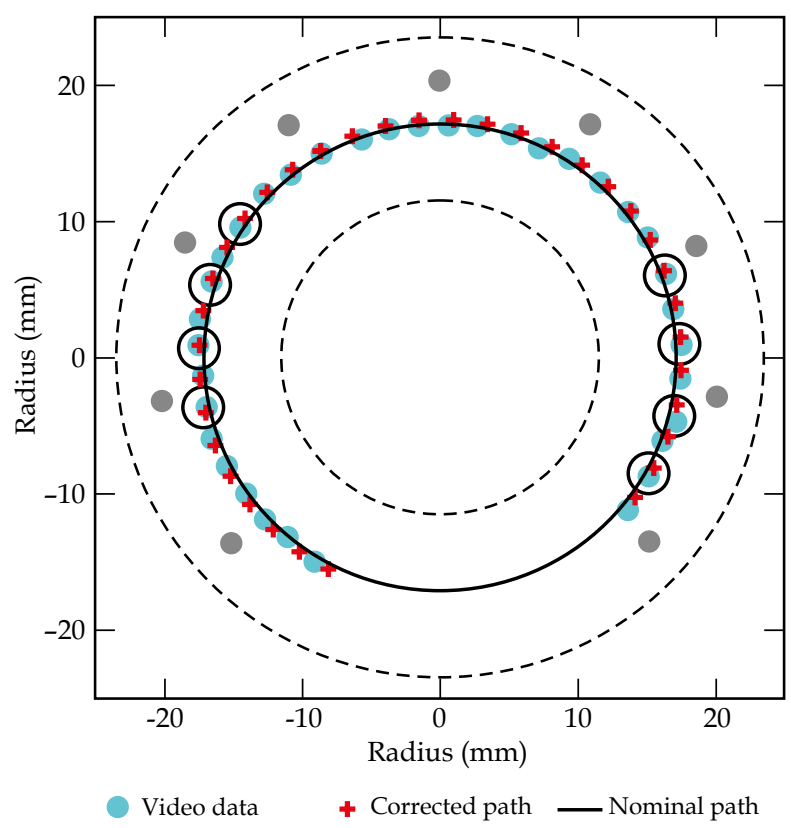

Fig. 1. Template for the R34 ring applicator, showing the inner and outer diameter (dashed lines), positions of the holes for interstitial needles (grey circles), dwell positions observed in video analysis (blue circles), positions predicted by the corrected source path model (red crosses), the nominal path (solid line), and most frequently active dwell positions (black circles) of a standard loading pattern loader devices push a source wire with the source capsule at its end into the applicator or pull it back from the most distal point with a fixed step size. While the source capsule step size between two dwell positions remains constant along a straight line, the source positions will not follow an exact circular motion as the source is moved through the applicator by the afterloader [7]. Nevertheless, as a first approximation, the source path inside the ring applicator can be described by a circle, i.e., the distance $(r)$ between the centre of the source and the centre of the ring, and the polar angle $(\varphi)$ between two consecutive dwell positions remain constant as the source is moved through the applicator by the afterloader.

The purpose of this study was to investigate the validity of the nominal circular source path model defined as the center of the source channel. To evaluate the impact of deviations of the source path from a circle on accurate dose planning, the exact motions of the sources inside three widely used CT- and MR-compatible plastic ring applicators (Vienna CT/MR ring applicators, Elekta AB, Sweden) were analysed. The results of this precise source path reconstruction were used to adjust the path shape for applicators in clinical use in the corresponding planning system. The validity of the nominal circular source path model as well as the dosimetric impact of source position uncertainties for treatment planning were investigated by simulating clinically relevant uncertainties in a set of patient treatment plans.

If non-negligible deviations exist, they must be minimized by adequate determination of the real source path during applicator commissioning. Therefore, as a part of this study, the potential of an autoradiograph-based method to adjust the circular source path model to better match the most clinically relevant dwell positions was tested.

\section{Material and methods}

\section{Video analysis}

Three CT-/MR-compatible Vienna ring applicators (Elekta AB, Sweden) with nominal diameters $d=26,30$ and $34 \mathrm{~mm}$ (R26, R30, and R34, hereafter) were investigated. In order to be able to reconstruct the real source path with high precision, a detailed video analysis of the motion of the source through the applicator channel was performed. For this purpose, the applicators were cut open and covered with a transparent plate (Figure 1 in [7]). This allowed to record the stepwise motion of the source through the ring during irradiation with a microSelectron ${ }^{\circledR}$ afterloader (Elekta AB, Sweden), with a digital camera mounted in front of the applicator. For each applicator, the selected dwell position sequence was repeated 10 times in order to evaluate possible random variations between individual irradiations. Each sequence included a maximum of 33 dwell positions distributed over the whole length of the ring. The dwell time for each position was set to $2.0 \mathrm{~s}$.

The obtained digital images were processed with the commercial software Adobe Photoshop ${ }^{\circledR}$ (Adobe Systems Inc., USA) and Graphmatica (kSoft, Inc., USA). A graphical template was generated from the applicator model of the treatment planning system (TPS). It included the nom- 
inal dwell coordinates extracted from Oncentra GYN v1.1 (Nucletron, Veenendaal, The Netherlands), as well as additional reference data, such as the inner and outer diameter of the investigated ring, and the positions of interstitial needles passing through the ring. An example of the template for a R34 ring is shown in Figure 1.

The digital images extracted from the video analysis of all three ring applicators were then superimposed with the respective templates. To evaluate the observed dwell positions, the coordinates were transferred from the Photoshop system to the reference frame of the graphical template. The maximum uncertainty introduced by this manual procedure was estimated to be $0.5 \mathrm{~mm}$, by multiple repetitions.

The differences between the observed dwell positions and coordinates derived by the TPS were determined. Differences are reported as the absolute distance $d$ between the two coordinates. In addition, the shifts are represented by angle deviations. These angle deviations can be recalculated to arc length deviations $d_{r}$ with the appropriate source path diameter. Based on this, \pm deviations are reported, which are related to clockwise and counterclockwise shifts.

To optimize the circular source path model, stored within the TPS, the source path radius was varied in an iterative procedure, using all the observed source positions, until a best fit to the experimental measurements was achieved.

\section{Autoradiography}

The final corrected source paths derived from the video analysis were then verified with autoradiography. For the radiographical analysis, the ring applicators were attached to Kodak ${ }^{\mathrm{TM}} \mathrm{X}$-Omat $\mathrm{V}^{\mathrm{TM}} \mathrm{X}$-ray films. For stable positioning of the applicators on the X-ray film, a custom built phantom was used. The films were irradiated by the microSelectron ${ }^{\circledR}$ afterloader, using a radiation sequence with four or more dwell positions for each applicator. All irradiations were performed with an air kerma rate $\sim 2000 \mathrm{cGy} \mathrm{cm}^{2} \mathrm{~h}^{-1}$ and dwell times between 0.8 and $2.0 \mathrm{~s}$. After irradiation with the $\operatorname{Ir}^{192}$ source, $X$-ray markers were inserted into the applicators, which remained attached to the films, and X-ray images were obtained $(90 \mathrm{kV}, 40 \mathrm{mAs}$, $110 \mathrm{~ms}$ exposure time). For each ring, a set of the most relevant dwell positions in accordance to the standard loading pattern (i.e. no. 1, 3, 13, 18, 23, 28, 33) were irradiated.

From the resulting images, the coordinates of the dwell positions were again measured using custom made templates. The radiography based coordinates were compared to the planned coordinates listed by the TPS and the results of the previous video analysis of the source path, in order to verify the compatibility of the two measurement techniques and to test the validity of the corrected source path model.

\section{Dosimetric evaluation}

An accurate localization of the source path leads to an increased accuracy in dose planning. In order to evaluate the effect of a dislocated source path on dose-volume histogram (DVH) parameters for targets and organs at risk, ten consecutive clinical plans for primary cervix brachytherapy computed with Oncentra GYN v1.1 were analysed using the new adjusted circular source path. Starting from the original individual loading patterns and dwell times for each patient, the source positions were rotated $\pm 2.5 \mathrm{~mm}$ and $\pm 5.0 \mathrm{~mm}$ clockwise/counter clockwise. Clinically relevant $\mathrm{DVH}$ parameters $\left(\mathrm{D}_{90}\right.$ for gross tumour volume [GTV] and clinical target volume $(\mathrm{CTV})_{\mathrm{HR}}, \mathrm{D}_{2 \mathrm{~cm}^{3}}$ for OARs) for the four additional, adapted plans, with rotations of the whole source path were analysed and compared to the DVHs of the original, clinically used plans. This simulates a systematic uncertainty of the whole source path rotation.

In addition to the overall rotation of the loading pattern by one or two dwell positions $( \pm 2.5 \mathrm{~mm}$ and $\pm 5.0 \mathrm{~mm})$, the differences in DVH parameters obtained from the original source path and the corrected source path model were investigated. Dosimetric evaluation of the same 10 clinical treatment plans was performed again, using the nominal source path diameter and the corrected source path with the increased radius.

\section{Results}

\section{Video analysis}

During the first stage of the video analysis, 8 dwell positions were observed 10 times in each ring. In a second sequence, all available dwell positions were observed three times each. But in comparison to the findings in the recently published paper from [5], no dwell position shifts between individual measurement sets performed with the same applicator type have been detected. Therefore, the source path correction was performed on the observation of one individual series including several available dwell positions, for each applicator. Individual video images were superimposed on the templates generated from the TPS coordinates.

The average distances between dwell coordinates given by the TPS using a circular pathway with nominal diameter and the video measurements were $3.6 \pm 1.3 \mathrm{~mm}$, $4.5 \pm 1.3 \mathrm{~mm}$, and $3.2 \pm 0.9 \mathrm{~mm}$ for R26, R30, and R34, respectively. Assuming a circular source path, the measurements correspond to the respective radii $13.5 \pm 0.2 \mathrm{~mm}$, $15.9 \pm 0.2 \mathrm{~mm}$, and $17.4 \pm 0.2 \mathrm{~mm}$ instead of the nominal source path radii of 13,15 , and $17 \mathrm{~mm}$ provided by the TPS. For a constant source step size, $s=2.5 \mathrm{~mm}$, an increased radius $r$ leads to a decreased angle a between two consecutive source positions, assuming the arc length $b$ to be equal to the step size $s$ and $b=\mathrm{r} \pi \alpha / 180$.

Consequently, the video analysis reveals an increase of the difference between observations and TPS dwell coordinates for higher dwell position numbers. This indicates that if one of the intermediate dwell positions (which are clinically relevant for standard loading patterns [6] remains fixed, and the radius $r$ is varied iteratively, an optimum radius that minimises the maximum of the differences between observed $\left(X_{\text {obs }}\right)$ and corrected $\left(X_{\text {corr }}\right)$ dwell positions $\left(d_{\max }\left(X_{o b s}-X_{\text {corr }}\right)\right)$, can be determined.

For the R34 applicator and a fixed dwell position no. 9, an average distance $\left(\bar{d}\left(X_{o b s}-X_{c o r r}\right) \pm 1 \sigma\right)$ between video 
measurements and the corrected dwell positions of 0.6 $\pm 0.3 \mathrm{~mm}$ was obtained with a corrected source path radius $r_{\text {corr }}=17.5 \mathrm{~mm}$. In this case, the maximum distance $\left(d_{\max }\left(X_{o b s}-X_{c o r r}\right)\right)$ was $1.3 \mathrm{~mm}$. The results of the source path correction for all 3 applicators are summarised in Table 1. An illustration of the corrected source path for the R34 applicator is shown in Figure 1. Deviations between observed and corrected dwell positions for the R34 ring are shown in Figure 2.

\section{Autoradiography}

The accuracy of the coordinate measurements on autoradiographs is influenced by geometrical distortions, e.g. caused by a $3 \mathrm{~mm}$ distance between the radiation source and the surface of the film, low contrast for short irradiation times, and the size and shape of the point spread

Table 1. Results of the iterative source path correction

\begin{tabular}{lccc} 
Applicator & R34 & R30 & R26 \\
\hline$r_{\text {TPS }}(\mathrm{mm})$ & 17 & 15 & 13 \\
\hline $\bar{d}\left(X_{\text {obs }}-X_{\text {TPS }}\right) \pm 1 \sigma(\mathrm{mm})$ & $3.2 \pm 0.9$ & $4.5 \pm 1.3$ & $3.6 \pm 1.3$ \\
\hline$d_{\text {max }}\left(X_{\text {obs }}-X_{\text {TPS }}\right)(\mathrm{mm})$ & 4.6 & 6.1 & 5.7 \\
\hline Fixed dwell position & 9 & 7 & 6 \\
\hline$r_{\text {corr }}(\mathrm{mm})$ & 17.50 & 15.85 & 13.95 \\
\hline $\bar{d}\left(X_{\text {obs }}-X_{\text {corr }}\right) \pm 1 \sigma(\mathrm{mm})$ & $0.6 \pm 0.3$ & $0.7 \pm 0.4$ & $0.8 \pm 0.5$ \\
\hline$d_{\text {max }}\left(X_{\text {obs }}-X_{\text {corr }}\right)(\mathrm{mm})$ & 1.3 & 1.3 & 2.0 \\
\hline $\bar{d}_{r}\left(X_{\text {obs }}-X_{\text {corr }}\right) \pm 1 \sigma(\mathrm{mm})$ & $0.1 \pm 0.7$ & $0.1 \pm 0.7$ & $-0.1 \pm 0.8$
\end{tabular}

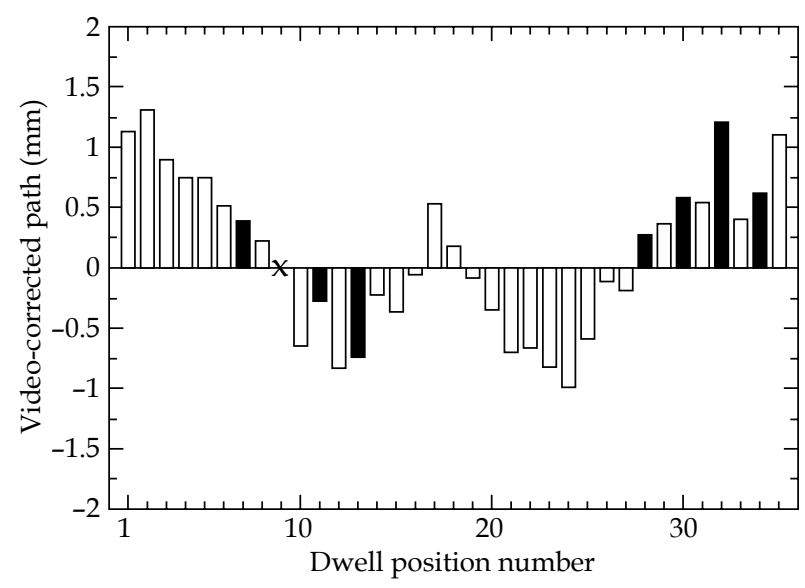

Fig. 2. Shifts $d_{r}$ projected along the source path between dwell positions derived from video analysis and those corresponding to the corrected source path model. Filled bars indicate the 8 most relevant positions for the R34 standard loading pattern. Dwell position no. 9, which was fixed during the iteration process, is marked with a cross

Table 2. Results from autoradiography measurements of the corrected circular source path

\begin{tabular}{lccc} 
Applicator & R34 & R30 & R26 \\
\hline $\bar{d}_{r}\left(X_{\text {obs }}-X_{\text {TPS }}\right)(\mathrm{mm})$ & $0.8 \pm 1.3$ & $0.1 \pm 1.5$ & $0.1 \pm 1.5$
\end{tabular}

function of the radiating (line) source on the X-ray image. The uncertainty for reconstruction of the dwell positions from radiographs is therefore of the order of the source step size. Hence, the full true source path could not be reconstructed precisely with this method. However, autoradiography was used to verify the quality of the final corrected source path model obtained by video analysis. In comparison with autoradiographs, the original circular source path with nominal diameter clearly shows large inconsistencies of the order of several millimetres. Displacements of $6 \mathrm{~mm}$ and more for several dwell positions indicated the strong need for source path corrections. After adjusting the path via video analysis, the average differences between the corrected dwell positions and those measured from radiographs are listed in Table 2 and show an average rotation shift $\left(\bar{d}_{r}\left(X_{o b s}-X_{T P S}\right)\right)$ of 0.1 to $0.8 \mathrm{~mm}$.

\section{Dosimetric evaluation}

The results of the dosimetric evaluation of dislocated dwell positions are shown in Table 3. In the case of a clockwise source path rotation by $2.5 \mathrm{~mm}$, i.e., the nominal distance between two dwell positions, an average variation of the doses for the most exposed $2 \mathrm{~cm}^{3}$ volumes $\left(\mathrm{D}_{2 \mathrm{~cm}^{3}}\right)$ by $2.6 \pm 2.7 \%, 1.3 \pm 1.9 \%$, and $0.2 \pm 2.3 \%$ was observed for rectum, bladder, and sigmoid, respectively. Rotating the loading pattern by one more dwell position, i.e., a total dislocation of source positions by $5.0 \mathrm{~mm}$ from the original setup, resulted in an average change of the $\mathrm{D}_{2 \mathrm{~cm}^{3}}$ for rectum and bladder by $6.7 \pm 5.1 \%$ and $3.2 \pm 3.6 \%$ respectively, while the average $\mathrm{D}_{2 \mathrm{~cm}^{3}}$ of the sigmoid varied only by 0.1 $\pm 4.2 \%$. The effect of source path rotations on the dose delivery to organs at risk was, as expected, most pronounced for the rectum. Large standard deviations were observed for all three investigated organs at risk. For target structures, i.e., $\mathrm{CTV}_{\mathrm{HR}}$ and $\mathrm{GTV}$, the $\mathrm{D}_{90}$ of the original plans were compared to the values corresponding to the four rotated loadings. For a clockwise rotation of $2.5 \mathrm{~mm}$, the $\mathrm{D}_{90}$ of the CTV $\mathrm{HR}_{\mathrm{H}}$ and GTV varied by $-0.5 \pm 1.3 \%$ and $-0.4 \pm 1.7 \%$, respectively. A rotation by two dwell positions in the same direction led to an average deviation of $\mathrm{D}_{90}$ $\mathrm{CTV}_{\mathrm{HR}}$ and GTV by $-1.2 \pm 2.9 \%$ and $-1.1 \pm 3.5 \%$.

These results are in agreement with a previous study of the effect of applicator shifts on dose distributions by Tanderup et al. [4].

Comparison of DVH parameters from treatment plans computed with the corrected source path model and those obtained from plans using the nominal source path revealed an inaccuracy, especially in the $D_{90}$ of the target volumes (5.5\% for $\mathrm{CTV}_{\mathrm{HR}}$ and $+0.2 \%$ for GTV), and also in $\mathrm{D}_{2 \mathrm{~cm}^{3}}$ of rectum and sigmoid. The $\mathrm{D}_{2 \mathrm{~cm}^{3}}$ of the bladder decreased by an average of $1.4 \%$. The largest deviation was found for the $\mathrm{D}_{90}$ of the $\mathrm{CTV}_{\mathrm{HR}}$, which ranged from $+5.5 \pm 5.9 \%$.

\section{Discussion and conclusion}

The correct source path definition is an essential part of applicator reconstruction for 3D image-based brachytherapy. It can be defined from the visible dummy wire in $\mathrm{CT}$, contrast material in tubes for MR imaging or 
Table 3. Ranges for average deviations (mean $\pm 1 \sigma$ ) for $D_{2 \mathrm{~cm}^{3}}$ of organs at risk and $D_{90}$ of targets caused by rotation of the loading pattern by one or two dwell positions in clockwise (+) or counter clockwise $(-)$ direction

\begin{tabular}{lcccc} 
Rotation & $-5.0 \mathrm{~mm}$ & $-2.5 \mathrm{~mm}$ & $+\mathbf{2 . 5} \mathbf{m m}$ & $+5.0 \mathrm{~mm}$ \\
\hline $\mathrm{D}_{2 \mathrm{~cm}^{3}}$ bladder & $(+0.65 \pm 5.25) \%$ & $(+0.15 \pm 2.65) \%$ & $(+1.30 \pm 1.90) \%$ & $(+3.15 \pm 3.55) \%$ \\
\hline $\mathrm{D}_{2 \mathrm{~cm}^{3}}$ rectum & $(-1.80 \pm 6.00) \%$ & $(-1.65 \pm 2.95) \%$ & $(+2.55 \pm 2.65) \%$ & $(+6.65 \pm 5.05) \%$ \\
\hline $\mathrm{D}_{2 \mathrm{~cm}^{3}}$ sigmoid & $(-0.05 \pm 3.55) \%$ & $(-0.35 \pm 1.95) \%$ & $(-0.20 \pm 2.30) \%$ & $(+0.05 \pm 4.15) \%$ \\
\hline $\mathrm{D}_{90} \mathrm{CTV}$ & $(+0.35 \pm 2.65) \%$ & $(+0.35 \pm 1.55) \%$ & $(-0.45 \pm 1.25) \%$ & $(-1.20 \pm 2.90) \%$ \\
\hline $\mathrm{D}_{90} \mathrm{GTV}$ & $(+0.30 \pm 3.20) \%$ & $(+0.25 \pm 1.65) \%$ & $(-0.40 \pm 1.70) \%$ & $(-1.10 \pm 3.50) \%$ \\
\hline
\end{tabular}

on radiographs, directly for each application or by using library plans derived from $\mathrm{CT}$ and/or radiographs $[3,6,8]$. Especially when applying MR for imaging, templates describing the source path in relation to visible parts of the applicator (like markers, holes, outer surface, and so on) are used $[7,9,10]$. An accurate source path definition is needed for these methods in particular.

In the source channel of a ring applicator, the source did not follow a circle with the nominal diameter given by the manufacturer. Reports on deviations have been published in literature $[3,8,11]$. By using video analysis, the source movement and consequently the source path, which describes the location of the dwell positions could be analysed in detail. In contrary to the findings from Awunor et al. [5], the dwell positioning was exactly the same for each measurement set performed with the same ring applicator type. The source path for the investigated type of ring is not a circle. However, it is possible to approximate the source path by a circle different to the nominal diameter of the ring. The diameter and consequently defined dwell positions can be verified by autoradiography in each department. During the acceptance test of each individual ring applicator, the correct parameters can be identified to approximate the source path by a template for manual or automatic applicator reconstruction. Obviously, autoradiography cannot match the accuracy of the video analysis performed in this study. However, verifications with autoradiographs showed that the uncertainty for the investigated type of applicator can be kept lower than $2 \mathrm{~mm}$ for $95 \%$ of the investigated positions, at least for the relevant source dwell positions. For target parameters, this is related to uncertainties in the range of $2 \%$, while $5 \%$ deviations are still possible for the rectum (within one standard deviation). For $5.0 \mathrm{~mm}$ source path shifts these deviations are about twice as high. Assuming a linear dependency, this results in uncertainties of $2 \%$ for a $1.0 \mathrm{~mm}$ shift of all dwell positions in the case of the rectum. Such accuracy is possible for the definition for the source path used in one single applicator by video analysis and assuming a circular source path with an adapted diameter.

The accurate source position should be provided by the vendor and has to be verified by autoradiography in each department. Accurate source path definition is essential in order to reduce dose deviations of clinical impact.

\section{Acknowledgements}

This work was supported by the Austrian Fonds zur Förderungwissenschaftlicher Forschung (FWF), project L-562.

\section{Disclosure}

The Department of Radiotherapy at the Medical University of Vienna receives financial and/or equipment support for research and educational purposes from Elekta $\mathrm{AB}$ and Varian Medical Systems Inc. C. Kirisits is a consultant to Elekta AB.

\section{References}

1. Tanderup K, Nesvacil N, Potter R et al. Uncertainties in image guided adaptive cervix cancer brachytherapy: impact on planning and prescription. Radiother Oncol 2014; 107: 1-5.

2. Kirisits C, Rivard MJ, Baltas D et al. Review of clinical brachytherapy uncertainties: analysis guidelines of GEC-ESTRO and the AAPM. Radiother Oncol 2014; 110: 199-212.

3. Hellebust TP, Kirisits C, Berger D et al. Recommendations from Gynaecological (GYN) GEC-ESTRO Working Group (III): considerations and pitfalls in commissioning and applicator reconstruction in 3D image-based treatment planning of cervix cancer brachytherapy. Radiother Oncol 2010; 96: 153-160.

4. Tanderup K, Hellebust TP, Lang S et al. Consequences of random and systematic reconstruction uncertainties in 3D image based brachytherapy in cervical cancer. Radiother Oncol 2008; 89: 156-163.

5. Awunor OA, Dixon B, Walker C. Direct reconstruction and associated uncertainties of 192Ir source dwell positions in ring applicators using gafchromic film in the treatment planning of HDR brachytherapy cervix patients. Phys Med Biol 2013; 58: 3207-3225.

6. Kirisits C, Pötter R, Lang S et al. Dose and volume parameters for MRI-based treatment planning in intracavitary brachytherapy for cervical cancer. Int J Radiat Oncol Biol Phys 2005; 62: 901-911.

7. Berger D, Dimopoulos J, Pötter R et al. Direct reconstruction of the Vienna applicator on MR images. Radiother Oncol 2009; 93: 347-351.

8. Hellebust TP, Tanderup K, Bergstrand ES et al. Reconstruction of a ring applicator using CT imaging: impact of the reconstruction method and applicator orientation. Phys Med Biol 2007; 52: 4893-4904.

9. Perez-Calatayud J, Kuipers F, Ballester F et al. Exclusive MRIbased tandem and colpostats reconstruction in gynaecological brachytherapy treatment planning. Radiother Oncol 2009; 91: 181-186.

10. Haack S, Nielsen SK, Lindegaard JC et al. Applicator reconstruction in MRI 3D image-based dose planning of brachytherapy for cervical cancer. Radiother Oncol 2009; 91: 187-193.

11. Varian Customer Bulletin Medical Device Correction - Field Safety Correction. PNL-GM-CR 302712008. 